\title{
The Combination of Antimicrobial Photodynamic Therapy and Photobiomodulation Therapy for the Treatment of Palatal Ulcers: A Case Report
}

\author{
Rafael Maya ${ }^{1}$, Lorena Lúcia Costa Ladeira ${ }^{2}$, Juliana Elaine Pinto Maya ${ }^{3}$, Letícia Machado Gonçalves ${ }^{4}$, Sandra \\ Kalil Bussadori ${ }^{5}$, Marco Aurélio Benini Paschoal ${ }^{*}$
}

${ }^{1}$ Florence Institute of Superior Teaching, São Luís - MA, Brazil

${ }^{2}$ Post Graduate Program in Public Health, Federal University of Maranhão - UFMA, São Luís - MA, Brazil

${ }^{3}$ Post Graduate Program in Pediatric Dentistry, São Leopoldo Mandic - SLMandic, Campinas - SP, Brazil

${ }^{4}$ Department of Dentistry I, Federal University of Maranhão - UFMA, Brazil

${ }_{5}^{5}$ Postgraduate Program in Biophotonics Applied to Health Sciences, Universidade Nove de Julho - UNINOVE, São Paulo SP, Brazil

${ }^{6}$ Department of Pediatric Dentistry and Orthodontics, Federal University of Minas Gerais - UFMG, Belo Horizonte - MG, Brazil

\section{*Correspondence to \\ Marco Aurelio Benini Paschoal, Email: \\ marcobpaschoal@hotmail.com}

Published online March 15 2020

\begin{abstract}
Introduction: One of the unexpected side effects of the Haas type palatal expander is ulcers progressing to necrotic lesions in the palatal area due to poor hygiene. The use of antibiotic therapy is mandatory. However, long periods of healing/pain and the need for a systemic host response with the aid of metabolization, especially in children, are issues that should be taken into account in the management of this type of injury. Since phototherapy modalities (antimicrobial photodynamic therapy [aPDT] and photobiomodulation therapy [PBMT]) are able to enhance and accelerate the healing process and reduce the bacterial load, this case report aimed to describe the use of the above-mentioned therapies to treat palatal ulcers occurring during orthodontic expansion.

Case Report: The patient, a 10-year-old boy, with a chief complaint of bleeding and continuous pain in the region of his expander was verified on a follow-up visit. After a dental examination, the expander was removed and two necrotic lesions which were in contact with the acrylic part of the tooth-tissue expander were found in the palatal region. The proposal was to use one aPDT session with methylene blue followed by 4 sessions of PBMT with a red laser diode. On the 5th day, reorganized tissue was verified, with the absence of bleeding, swelling, and pain. On the 20th day of follow-up, the area showed no signs of inflammation, healthy tissue without any pathological clinical symptoms, and complete wound healing.

Conclusion: The concomitant use of PBMT and APDT therapies may be considered feasible as an adjunct treatment to manage palatal ulcers resulting from the incorrect use of tooth-tissue types of expanders.
\end{abstract}

Keywords: Low-level laser therapy; Oral ulcer; Palatal expansion technique.

\section{Introduction}

Some occlusal discrepancies are based on the use of fixed tooth-borne (Hyrax-type expander) and tooth-tissueborne devices (such as the Haas-type expander) for the correction of posterior crossbite malocclusion. ${ }^{1}$ The difference between the tooth-borne and the tooth-tissueborne types of appliances is that the latter appliance has acrylic plates that cover the palatal mucosa bilaterally and is screw-retained with an $11-\mathrm{mm}$ screw. ${ }^{2}$

When the tooth-tissue-borne type of expander is used, the ulceration of the palatal mucosa may occur due to the acrylic part. In the presence of ulcers, symptoms such as swelling and pain can be present. ${ }^{3}$ When this occurs, the most common approach is to remove the device and prescribe anti-inflammatory/analgesic medication. In addition, when an infection is present (e.g. excessive bleeding, fever, and pain), the concomitant use of antibiotics is mandatory. Since the use of this therapy is capable of causing some side effects, adjunct methods, including photobiomodulation therapy, (PBMT) become a feasible "local biological" option because of reducing the painful symptomatology and modulating the inflammatory process, and when associated with some specific dyes (known as antimicrobial photodynamic

Please cite this article as follows: Maya R, Ladeira LLC, Maya JEP, Gonçalves LM, Bussadori SK, Paschoal MAB. The combination of antimicrobial photodynamic therapy and photobiomodulation therapy for the treatment of palatal ulcers: a case report. J Lasers Med Sci. 2020;11(2):228-233. doi:10.34172/jlms.2020.38. 
therapy - aPDT), it is capable of microbial eradication/ control, facilitating wound healing and the recovery of the patient's well-being. ${ }^{4,5}$

Therefore, the present study reports a clinical case of an approach to palatal ulcers in a young child during the fixed tooth-tissue borne palatal disjunction in combination with different photobiomodulation modalities based on the use of lasers.

\section{Case Report}

During the visit of the patient, a 10-year-old boy, for following up his Haas expander-type appliance at a private Dental School in São Luís - MA, Brazil, he presented the main complaints of bleeding, fetid odor, and a sensation of fever associated with painful symptomatology showing a very unwell condition. After the meticulous verification of the dental and medical history, which revealed no systemic disease, the device was removed revealing the presence of two ulcers in the palatal region, covered by a pseudomembrane that consisted of white fibrinoleukocyter tissue (Figure 1A).

After removing the appliance, the area was disinfected with $0.9 \%$ sterile saline solution and chlorhexidine $2 \%$ followed by one aPDT-session with methylene blue $0.005 \%$ $(0.0075 \% \mathrm{w} / \mathrm{v})$ solution (Chimiolux, 5\%, DMC, São Carlos - SP, Brazil), supported by sterile cotton dressings on both lesions. The aim was to reduce the bacterial load locally and to facilitate/accelerate the healing process. The dye remained in place for 5 minutes (pre-irradiation time - PIT) and was irradiated with a GaAlAs diode lowlevel laser (Thera Lase EC, DMC, São Carlos - SP, Brazil) onto only one point delivering $1 \mathrm{~J}$ of energy and $35 \mathrm{~J} /$ $\mathrm{cm}^{2}$ of fluence. Other laser specifications and irradiation protocols are described in Table $1 .^{6-8}$ Concomitantly, as a gold standard approach following specific guidelines, ${ }^{9,10}$ an antibiotic was prescribed to be taken every 8 hours (Amoxicillin, 500 mg, Merck, São Paulo - SP, Brazil) for 7 days due to the high level of infection, bleeding, discomfort, signs of systemic dissemination of infection (prostration and a sensation of fever), and avoiding the onset of opportunistic infections.

After this, the patient was re-called within 48 hours to verify his clinical condition and we proceeded with the application of PBMT with the same parameters as those shown above to 4 distinct points (around the lesions at points equally far from each other) according to the details described in Table 1, as used in some previous studies (Figure 1B). ${ }^{11-14}$ The irradiation was repeated 4 times with an interval of 48 hours; therefore, 4 PBMT sessions were performed with the aim of relieving pain (analgesic effect), controlling inflammation, and accelerating the healing process with the main focus being on patient comfort.

The clinical condition was evaluated after 5 days (Figure 1C), showing a reduction in the lesion, the control of the inflammatory process with more organized tissue, hemostasis, very good coagulation and the absence of pain reported by the patient. On the $20^{\text {th }}$ day of follow-up, a total remission of the lesions with a high level of healthy tissue accompanied by the lack of pain constituted the most favorable aspects (Figure 1D).

After complete healing, the patient was recalled and the orthodontic therapy continued, as requested by the patient's family and the professionals responsible for the case. Both the guardians and the patient were provided with recommendations regarding oral care (and hygiene

Table 1. Technical Parameters for the Reported Case

\begin{tabular}{|c|c|c|}
\hline & Technical Parameters & \\
\hline & aPDT & РВMT \\
\hline Light source & Low-level diode laser & \\
\hline Active medium & GaAlAs & \\
\hline Wavelength & $660 \mathrm{~nm} \pm 10 \mathrm{~nm}$ & \\
\hline Power output & $0.1 \mathrm{~W}( \pm 20 \%), \mathrm{CW}$ & \\
\hline Irradiation mode and frequency of treatment & $\begin{array}{l}\text { Contact, punctual in } 1 \text { day (unique } \\
\text { application) }\end{array}$ & $\begin{array}{l}\text { Contact, punctual in } 4 \text { days (unique } \\
\text { applications on noncoconsecutive days) }\end{array}$ \\
\hline Model / Manufacturer & Thera Lase EC/DMC, São Carlos - SP, Brazil & \\
\hline Spot area & $0.94 \mathrm{~cm}^{2}$ & \\
\hline Exposure time per point/session/time treatment per week & $10 \mathrm{~s}$ & $10 \mathrm{~s} / 40 \mathrm{~s} / 120 \mathrm{~s}$ \\
\hline Number of points & 1 & 4 \\
\hline Energy per point & $1 \mathrm{~J}$ & \\
\hline Energy per daily session & $1 \mathrm{~J}$ & 4J \\
\hline Dye/PIT & Methylene blue $0.05 \% / 5 \mathrm{~min}$ & Not applicable \\
\hline Fluence per point & $35 \mathrm{~J} / \mathrm{cm}^{2}$ & \\
\hline Total fluence in each session & $35 \mathrm{~J} / \mathrm{cm}^{2}$ & $140 \mathrm{~J} / \mathrm{cm}^{2}$ \\
\hline Irradiance per point & $0.094 \mathrm{~W} / \mathrm{cm}^{2}$ & $0.376 \mathrm{~W} / \mathrm{cm}^{2}$ \\
\hline Cumulative dose given & $595 \mathrm{~J} / \mathrm{cm}^{2}$ & \\
\hline Total irradiation time & $170 \mathrm{~s}$ & \\
\hline
\end{tabular}

Abbreviations: aPDT, antimicrobial photodynamic therapy; PBMT, photobiomodulation therapy; CW, continuous wave; PIT, pre-irradiation time. 

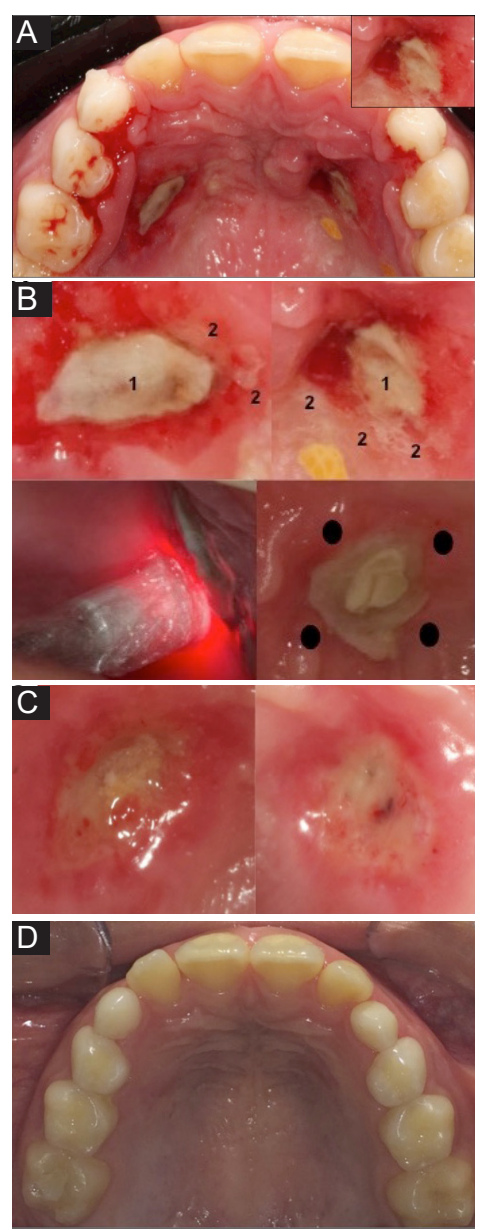

Figure 1. A. Initial aspect of necrotic lesions. In the upper right side, a close view of lesion. B. Upper left and right side: detailed view of necrotic lesions. 1. White necrotic pseudomembrane; 2. Necrotic areas surrounded by bleeding channels. Lower left side: $1^{\text {st }}$ application of PBMT session after 48 hours. Lower right side: Application points of PBMT around necrotic pseudomembrane. C. Aspect of necrotic lesions after 5 days of follow-up. D. Aspect of necrotic lesions after 20 days of follow-up.

as well) during the use of the appliance. However, instead of using the Haas-type expander, this second palatal expansion was performed with a fixed tooth-borne device (Hyrax-type expander).

\section{Discussion}

The present case report demonstrated an innovative and adjunct approach, in which both phototherapy (aPDT and PBMT) modalities were chosen to enhance the healing process and reduce the bacterial load in necrotic lesions, with the aim of providing patient comfort and reducing the time for the recovery of the ulcerative palatal region.

In the case reported here, the Haas-type expander was selected due to its maximum anchorage that provided stable expansion. ${ }^{15,16}$ In addition, apart from the type of expander, the biochemical reaction to expansion includes the cardinal signs and symptoms of inflammation, such as swelling and pain, ${ }^{17}$ as verified in the present study. However, the presence of the acrylic part may lead to ischemia with the formation of necrotic lesions followed by pain, the ulceration of palatal mucosa and excessive bleeding. ${ }^{3}$

In a recent study, ${ }^{18}$ the authors revealed that pain was reported by Haas users, whereas no symptomatic effects were verified by individuals using Hyrax appliances. However, both types of expanders have been responsible for infection with the main complaint of difficulty during brushing and flossing, especially in the posterior region of the fornix, a site where food is compacted due to the natural movement of the tongue during mastication. Furthermore, other complications have been related, including persistent pain, tooth darkening, and asymmetric expansion for Haas users. Nevertheless, relative to which device would be less harmful to periodontal tissues, a current systematic review concluded that there were no significant differences between the two expanders and considered the devices equally safe for oral health. ${ }^{19}$

The proposed approach was firstly based on the gold standard protocol ${ }^{9,10}$ by means of antibiotic therapy (amoxicillin) since the open ulcers served as a microbial reservoir, with the possibility of blood contamination. Moreover, the symptomatology presented by the patient (fever state, painful, prostration and very unwell condition) showed the clear signs of disseminated infection, making prescription mandatory in such cases. Therefore, a broad-spectrum antibiotic was needed to avoid other infections, facilitate wound healing and promote the suitable well-being of the child. Furthermore, to support the decontamination of the oral condition locally, accelerate the healing process, and reduce the pain sensation, the laser device (in an infrared wavelength) was first used, associated with methylene blue (Chimiolux, 5\%, DMC, São Carlos - SP, Brazil) to constitute the aPDT modality. The parameters used, such as PIT (5 minutes), the use of a phenotiazine dye (methylene blue), the time of irradiation and dose, were in accordance with those of other investigations ${ }^{11-14}$ and are described in Table 1.

The use of aPDT is well documented for many diseases and conditions such as infected leg ulcers, ${ }^{20}$ diabetic foot, ${ }^{21}$ and chronic skin ulcers. ${ }^{22}$ With specific regard to the oral environment, its action includes the treatment of leukoplakia, ${ }^{17}$ lichen planus, ${ }^{23}$ periodontal pockets, ${ }^{24}$ peri-implantitis, ${ }^{25}$ and oral decontamination as well. ${ }^{26,27}$ In the specific case of palatal ulcers during orthodontic therapy, this is the first case report to use this approach to solve this type of injury. Other studies have reported that the use of aPDT accelerated wound healing in oral mucosa right after the second day in comparison with the control group and the group with the laser applied alone. ${ }^{7}$ In agreement with this finding, Silva et $\mathrm{al}^{28}$ verified that the rat groups submitted to aPDT (with phthalocyaninederived photosensitizer) applied in a gel base had a higher collagen content and enhanced re-epithelization when compared with the control group. Moreover, connective tissue remodeling was more evident in those specific groups. 
Recently, Nesi-Reis et $\mathrm{al}^{29}$ in a systematic review, researched articles that used aPDT for treating skin wounds in humans. They concluded that aPDT contributed to wound healing in several ways, such as leading to cellular death, reducing or increasing inflammation, fibroblast proliferation, collagen and elastin, and raising growth factor beta and metalloproteinases.

The aim of applying photodynamic therapy (aPDT) previously to PBMT, after dye application (methylene blue) in combination with an appropriate wavelength of visible light (preferred $\sim 660 \mathrm{~nm} /$ red light) was to form free radicals or superoxide ions (formed by type I - electron transference or type II - oxygen singlet reactive species) that oxidize the biological structures of bacteria/fungi/viruses (e.g., mitochondria, DNA, lipid membranes) causing a lethal photochemical reaction leading to irreversible damage with the consequent death of microorganisms. With this "microorganism biological cleaning" the contaminated area is capable of healing in a faster way in a short period of time, as shown by the clinical parameters obtained in the case reported here. . $^{30,31}$

PBMT sessions focused on biostimulating many types of cells and acts, especially in the palatal wound healing process, angiogenesis and remodeling by increasing collagen production after the bacterial disinfection provided by photodynamic therapy, preferably with a visible wavelength at $\sim 660 \mathrm{~nm} / \mathrm{red}$ light (Figure 1C). Moreover, PBMT is a non-invasive method, providing pain relief by analgesic and anti-inflammatory effects. ${ }^{4,32}$ PBMT can be performed with various wavelengths and units with different outputs. Furthermore, the analgesic effect has been suggested to occur by the production of neuropharmacological substances, including endogenous endorphins ( $\beta$-endorphin), a decrease in the activity of C-fibers and bradykinin, and altering the pain threshold. ${ }^{4}$ In addition, PBMT is able to improve the microcirculation/oxygenation of the tissue and stimulate epithelial, endothelial and mesenchymal cell growth. ${ }^{33}$ In the present case, a red wavelength GaAlAs laser ( $n=660$ $\mathrm{nm} \pm 10 \mathrm{~nm}$ ) was applied with $1 \mathrm{~J}$ of energy, dosimetry of $35 \mathrm{~J} / \mathrm{cm}^{2}$, contact in a continuous mode, 10 seconds of irradiation and a output power of $0.1 \mathrm{~W}$ divided into 4 application points around the wound equally far from each other (Figure 1). For this purpose, the spot size used was $0.94 \mathrm{~cm}^{2}$, delivering $0.094 \mathrm{~W} / \mathrm{cm}^{2}$ of irradiance per point; therefore, in total (4 points per session), it delivered $0.376 \mathrm{~W} / \mathrm{cm}^{2}$ of irradiance with a total dose of $595 \mathrm{~J} /$ $\mathrm{cm}^{2}$ given after the 4 sessions. All other parameters are described in Table 1. Comparing this specific parameter with those of other studies was difficult due to different laser types, the ways of application (contact, noncontact, continuous, pulsed), and the number of sessions, specific to each case.

Relative to protocols, the number of wound healing sessions varied among studies. Ahad et $\mathrm{al}^{34}$ used 5 sessions (10 days) to reach a substantial effect on a self-inflicted thermal injury in the palate by using a $1 \mathrm{~W}$ output laser, whereas da Silva Neves et $\mathrm{a}^{35}$ used a higher dosage $(60 \mathrm{~J} /$ $\mathrm{cm}^{2}$ ) with $0.3 \mathrm{~W}$ applied in 7 sessions to promote faster wound healing after the removal of a connective tissue graft in the palatal region. Comparing these protocols with those used in the present case was difficult. However, after 4 applications of PBMT with the support of 1 aPDT session, the first signs of tissue reorganization were verified on the $5^{\text {th }}$ day and total remission on the $20^{\text {th }}$ day. In general, injuries of this nature cause moderate to intense pain and heal within 7-10 days. However, since the lesions verified here were considered a larger type (greater than $5 \mathrm{~mm}$ in diameter), the wound generally heals in a period of up to 6 weeks. ${ }^{36}$ Therefore, comparing the present results with those in the literature, it was possible to verify the faster rate of recovery in the present case, in which both phototherapy modalities were applied. The differences observed were due to the area affected, lesion diameter and high level of infection, delaying the total healing process.

Furthermore, drawing meaningful comparisons with the parameters used and those found in the literature was a difficult task since the clinical outcomes depend on the adjustment of many variables (e.g. fluence, wavelength, output power, the time and mode of application, and the distance of the source from irradiated tissue resulting in different host responses).

When compared with antibiotic therapy used alone, ${ }^{10}$ the present clinical results demonstrated a substantial relief from the painful sensation in a short period of time, achieving healthy tissue marked by the absence of bleeding in few days and more organized wound healing, which resulted from the combination with/activation by light and protocols proposed here..$^{26,27,34,35}$ In the present report, no pain scale measurement was applied (e.g. visual analogic scale). However, immediately after removing the device, cleaning, and aPDT application, we found that the painful sensation had decreased on the second day (first PBMT session) by means of the patient's report. In a study by Akerzoul et al, ${ }^{36}$ a patient reporting recurrent aphthous ulcers showed pain relief right after the PBMT application, which is in agreement with a current systematic review that demonstrated significant pain relief immediately in five out of six studies. ${ }^{37}$

The limitation of this study was that it was performed in a single-patient case observation. The effects of the two treatment modalities could perhaps have overlapped, promoting a very good healing process and producing effects on the patient. Furthermore, it would not have been reasonable to try and infer or speculate distinct/separate effects of the two approaches (systemic - antibiotics and PBMT and local - aPDT) since they act together with the aim of providing comfort and a fast recovery, as verified here (less than a month) with no need for other drugs (e.g. analgesic and anti-inflammatory agents). Hence, further prospective studies are necessary to confirm these 
observations and optimize the clinical treatment of this injury.

\section{Conclusion}

In summary, the reported case demonstrated the benefits of the association of photobiomodulation modalities for the treatment of palatal ulcers during maxillary expansion. In addition, it provided an adjunct treatment that must be further investigated to attest to its efficacy and safety for use in young children.

\section{Ethical Considerations}

Informed consent was obtained from the patient for the publication of this report.

\section{Conflict of Interests}

The authors declare no conflict of interest.

\section{References}

1. Mundstock KS, Barreto G, Meloti AF, Araújo MA, dos Santos-Pinto A, Raveli DB. Rapid maxillary expansion with the Hyrax appliance: an occlusal radiographic evaluation study. World J Orthod. 2007;8(3):277-84.

2. Rodrigues AP, Monini AC, Jr Gandini LG, Santos-Pinto A. Rapid palatal expansion: a comparison of two appliances. Braz Oral Res. 2012;26(3):242-8. doi:10.1590/s180683242012000300010.

3. Handelman CS, Wang L, BeGole EA, Haas AJ. Nonsurgical rapid maxillary expansion in adults: report on 47 cases using the Haas expander. Angle Orthod. 2000;70(2):129-44. doi: 10.1043/0003-3219(2000)070<0129: nrmeia>2.0.co;2.

4. Silveira PC, Silva LA, Freitas TP, Latini A, Pinho RA. Effects of low-power laser irradiation (LPLI) at different wave-lengths and doses on oxidative stress and fibrogenesis parameters in an animal model of wound healing. Lasers Med Sci. 2011;26(1):125-31. doi: 10.1007/s10103-0100839-0.

5. Biel MA. Photodynamic therapy of bacterial and fungal biofilm infections. In: Gomer CJ, editor. Photodynamic therapy: Methods and protocols. Totowa NJ: Humana Press; 2010. p.175-94. doi: 10.1007/978-1-60761-697-9_13.

6. Sperandio FF, Simões A, Aranha AC, Corrêa L, Orsini Machado de Sousa SC. Photodynamic therapy mediated by methylene blue dye in wound healing. Photomed Laser Surg. 2010;28(5):581-87. doi: 10.1089/pho.2009.2601.

7. Deyhimi P, Khademi H, Birang R, Akhoondzadeh M. Histological evaluation of wound healing process after photodynamic therapy of rat oral mucosal ulcer. J Dent (Shiraz). 2016;17(1):43-8.

8. de Melo MCF, Silva de Macedo T, Biserra JA, Rodrigues KLC, Cruz M, Vidal AKL. The use of antimicrobial photodynamic therapy in oral injuries of a pediatric patient with myelodysplastic syndrome: case report. Spec Care Dentist. 2018;38(2):95-8. doi: 10.1111/scd.12275.

9. Scully AC, Boynton JR. Antibiotics and local anesthetics: Drug considerations for the child patient. J Mich Dent Assoc. 2017;99(2):36-41, 71.

10. Crawford J. Orofacial Infections and Antibiotic Management. In. Newman MG, Kornman KS, editors.
Antibiotic / Antimicrobial use in dental practice. ist ed. Berlin: Quintessence; 1990. p.22-30.

11. Min PK, Goo BL. $830 \mathrm{~nm}$ light-emitting diode low level light therapy (LED-LLLT) enhances wound healing: a preliminary study. Laser Ther. 2013;22(1):43-9. doi: 10.5978/islsm.13-OR-06.

12. Pereira PR, de Paula JB, Cielinski J, Pilonetto M, Von Bahten LC. Effects of low intensity laser in in vitro bacterial culture and in vivo infected wounds. Rev Col Bras Cir. 2014;41(1):49-55. doi: 10.1590/s0100-69912014000100010.

13. de Alencar Fonseca Santos J, Campelo MBD, de Oliveira RA, Nicolau RA, Rezende VEA, Arisawa EÂL. Effects of low-power Light therapy on the tissue repair process of chronic wounds in Diabetic Feet. Photomed Laser Surg. 2018;36(6):298-304. doi: 10.1089/ pho.2018.4455.

14. Brauncajs $M$, Ksiąszczyk $K$, Lewandowska-Polak A, Gorzela K, Grzegorczyk J. Impact of low-level laser therapy on the dynamics of pressure ulcer-induced changes considering an infectious agent and cathelicidin LL-37 concentration: a preliminary study. Postepy Dermatol Alergol. 2018;35(6):582-6. doi: 10.5114/ada.2018.77609.

15. Liu SS, Opperman LA, Kyung HM, Buschang PH. Is there an optimal force level for sutural expansion? Am J Orthod Dentofacial Orthop 2011;139(4):446-55. doi: 10.1016/j. ajodo.2009.03.056.

16. Gregório L, de Medeiros Alves AC, de Almeida AM, Naveda R, Janson G, Garib D. Cephalometric evaluation of rapid and slow maxillary expansion in patients with BCLP: Secondary data analysis from a randomized clinical trial. Angle Orthod. 2019;89(4):583-9. doi: 10.2319/081018589.1.

17. Li Y, Wang B, Zheng S, He Y. Photodynamic therapy in the treatment of oral leukoplakia: a systematic review. Photodiagnosis Photodyn Ther. 2010;25:17-22. doi: 10.1016/j.pdpdt.2018.10.023.

18. Pereira MD, Koga AF, Prado GPR, Ferreira LM. Complications from surgically assisted rapid maxillary expansion with Haas and Hyrax expanders. J Craniofac Surg. 2018;29(2):275-8. doi: 10.1097/SCS.0000000000004079.

19. Bastos RTDRM, Blagitz MN, Aragón MLSC, Maia LC, Normando D. Periodontal side effects of rapid and slow maxillary expansion: a systematic review. Angle Orthodontist. 2019;89(4):651-60. doi: 10.2319/060218419.1 .

20. Kawczyk-Krupka A, Pucelik B, Międzybrodzka A, Sieroń AR, Dąbrowski JM. Photodynamic therapy as an alternative to antibiotic therapy for the treatment of infected leg ulcers. Photodiagnosis Photodyn Ther. 2018;23:132-43. doi: 10.1016/j.pdpdt.2018.05.001.

21. Tardivo JP, Serrano R, Zimmermann LM, Matos LL, Baptista MS, Pinhal MAS, et al. Is surgical debridement necessary in the diabetic foot treated with photodynamictherapy? Diabet Foot Ankle. 2017;8(1):1373552. doi: 10.1080/2000625X.2017.1373552.

22. Reinhard A, Sandborn WJ, Melhem H, Bolotine L, Chamaillard M, Peyrin-Biroulet L. Photodynamic therapy as a new treatment modality for inflammatory and infectious conditions. Expert Rev Clin Immunol. 2015;11(5):637-57. doi: 10.1586/1744666X.2015.1032256.

23. Akram Z, Javed F, Hosein M, Al-Qahtani MA, Alshehri 
F, Alzahrani AL, Vohra F. Photodynamic therapy in the treatment of symptomatic oral lichen planus: a systematic review. Photodermatol Photoimmunol Photomed. 2018;34(3):167-74. doi: 10.1111/phpp.12371.

24. Akram Z. How effective is adjunctive antimicrobial photodynamic therapy in treating deep periodontal pockets in periodontal disease? A systematic review. J Investig Clin Dent. 2018;9(4):e12345. doi: 10.1111/jicd.12345.

25. Sivaramakrishnan G, Sridharan K. Photodynamic therapy for the treatment of peri-implant diseases: A network meta-analysis of randomized controlled trials. Photodiagnosis Photodyn Ther. 2018;21:1-9. doi: 10.1016/j. pdpdt.2017.10.013.

26. Santezi C, Reina BD, Dovigo LN. Curcumin-mediated Photodynamic Therapy for the treatment of oral infections - A review. Photodiagnosis Photodyn Ther. 2018;21:409-15. doi: 10.1016/j.pdpdt.2018.01.016.

27. Ricci Donato HA, Pratavieira S, Grecco C, Brugnera-Júnior A, Bagnato VS, Kurachi C. Clinical Comparison of two photosensitizers for oral cavity decontamination. Photomed Laser Surg. 2017;35(2):105-10. doi: 10.1089/pho.2016.4114.

28. Silva JC, Lacava ZG, Kuckelhaus S, Silva LP, Neto LF, Sauro EE, et al. Evaluation of the use of low-level laser and photosensitizer drugs in healing. Lasers Surg Med. 2004;34(5):451-7. doi: 10.1002/lsm.20062.

29. Nesi-Reis V, Lera-Nonose DSSL, Oyama J, Silva-Lalucci MPP, Demarchi IG, Aristides SMA, et al. Contribution of photodynamic therapy in wound healing: a systematic review. Photodiagnosis Photodyn Ther. 2018;21:294-305. doi: 10.1016/j.pdpdt.2017.12.015.

30. Dougherty TJ. An update on photodynamic therapy applications. J Clin Laser Med Surg. 2002;20(1):3-7. doi:
10.1089/104454702753474931.

31. Bevilacqua IM, Nicolau RA, Khouri S, Brugnera A Jr, Teodoro GR, Zângaro RA, et al. The impact of photodynamic therapy on the viability of Streptococcus mutans in a planktonic culture. Photomed Laser Surg. 2007;25(6):513-8. doi: 10.1089/pho.2007.2109.

32. Artés-Ribas M, Arnabat-Dominguez J, Puigdollers A. Analgesic effect of a low-level laser therapy $(830 \mathrm{~nm})$ in early orthodontic treatment. Lasers Med Sci. 2013;28(1):335-41. doi: 10.1007/s10103-012-1135-y.

33. Hagiwara S, Iwasaka H, Okuda K, Noguchi T. GaAlAs $(830 \mathrm{~nm})$ low-level laser enhances peripheral endogenous opioid analgesia in rats. Lasers Surg Med. 2007;39(10);797802. doi: 10.1002/lsm.20583.

34. Ahad A, Tasneem S, Lamba AK, Khan S. Healing of selfinflicted thermal injury of palatal mucosa by low-level laser therapy. Spec Care Dentist. 2017;37(6):314-7. doi: 10.1111/ scd.12256.

35. da Silva Neves FL, Silveira CA, Dias SB, Santamaria Júnior M, de Marco AC, Kerbauy WD, et al. Comparison of two power densities on the healing of palatal wounds after connective tissue graft removal: randomized clinical trial. Lasers Med Sci. 2016;31(7):1371-8. doi: 10.1007/s10103016-1988-6.

36. Akerzoul N, Chbicheb S. Low laser therapy as an effective treatment of recurrent aphtous ulcers: a clinical case reporting two locations. Pan Afr Med J. 2018; 30:205. doi: 10.11604/pamj.2018.30.205.15779.

37. Suter VGA, Sjölund S, Bornstein MM. Effect of laser on pain relief and wound healing of recurrent aphthous stomatitis: a systematic review. Lasers Med Sci. 2017;32(4):953-63. doi: 10.1007/s10103-017-2184-z. 